\title{
唾液酸衍生物的合成进展
}

\author{
王汝一 ${ }^{a} \quad$ 张 姝 ${ }^{b}$ 谭艳红 $^{a}$ 洪伟耀 ${ }^{a}$ 成 波 ${ }^{a}$

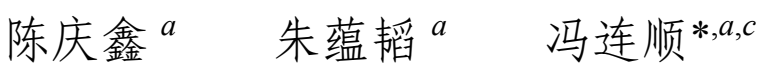 \\ $\left({ }^{a}\right.$ 北京大学化学与分子工程学院 北京 100871) \\ ${ }^{b}$ 谱尼测试科技股份有限公司 北京 100871) \\ ( ${ }^{c}$ 北京大学深圳研究生院化学生物学与生物技术学院 深圳 518055)
}

\begin{abstract}
摘要 唾液酸是一类九碳糖, 主要以糖脂或者糖蛋白的形式广泛分布于哺乳动物组织中, 是脊椎动物细胞表面最重要 的单糖. 唾液酸可修饰的位点较多, 目前已发展出成千上万的非天然唾液酸衍生物用于分子成像. 尽管如此, 非天然 糖代谢工程方法还处于发展的初期, 很多方面亟待发展和完善. 结合本课题组的研究成果, 着重介绍了唾液酸衍生物 的合成进展.
\end{abstract}

关键词 唾液酸; 合成; 进展

\section{Progress in the Synthesis of Sialic Acid Derivatives}

\author{
Wang, Ruyi ${ }^{a} \quad$ Zhang, Shu ${ }^{b}$ \\ Tan, Yanhong ${ }^{a}$ \\ Hong, Weiyao ${ }^{a}$ \\ Cheng, $\mathrm{Bo}^{a}$ \\ Chen, Qingxin ${ }^{a}$ \\ Zhu, Yuntao ${ }^{a}$ \\ Feng, Lianshun ${ }^{*, a, c}$
}

$\left({ }^{a}\right.$ Beijing National Laboratory for Molecular Sciences, Department of Chemical Biology, College of Chemistry and Molecular Engineering, Peking University, Beijing 100871)

$\left({ }^{b}\right.$ Pony Testing Co., Ltd., Beijing 100080)

( ${ }^{c}$ School of Chemical Biology and Biotechnology, Shenzhen Graduate School of Peking University, Shenzhen 518055)

\begin{abstract}
Sialic acids, a family of more than 50 members of $\alpha$-keto acidic monosaccharides with a 9-carbon backbone, are widely distributed in mammalian organisms in the form of glycolipids or glycoproteins. It is the most important monosaccharide appeared on the surface of cells of vertebrates. Nowadays, thousands of unnatural sialic acid derivatives have been synthesized for molecular imaging due to various modifiable sites in the sialic acid structure. However, metabolic oligosaccharide engineering (MOE), is still locating at its preliminary stage and requiring perfection urgently. Here, the research progress in the synthesis of sialic acid derivatives is reviewed.
\end{abstract}

Keywords sialic acid; synthesis; progress

唾液酸(Scheme 1)位于细胞膜糖蛋白质侧链末端, 通过 C-2 位异头碳的羟基以 $\alpha$-糖苷键连接在糖蛋白、糖 脂和寡糖的末端, 是细胞膜表面受体的重要组成部分, 是人体免疫系统中聚糖最为重要的组成部分之一. 大量 的研究表明唾液酸具有重要的生物学功能, 在抗炎、抗 肿瘤、抗病毒、抗老年痴呆等领域发挥着极为重要的作 用 ${ }^{[1 \sim 6]}$. 例如: 血清唾液酸水平明显升高是原发性肝癌 的标志之一, 检测血清唾液酸水平对原发性肝癌和肝硬 化的诊断有重要意义 ${ }^{[7]}$; 介导细胞表面黏附和信号传导 的选凝素(selectin)配体为包含唾液酸路易斯 X (sialyl lewis X)四糖的糖缀合物. 一些研究显示, 在许多肿瘤 细胞表面, 如肺癌细胞和黑色素瘤细胞某些含有唾液酸 的糖脂分子被过分表达，但是在正常组织中它们的含量 很少或者几乎不表达. 此外, 它直接参与白细胞迁移 (leukocyte migration)、B 细胞信号传导和激活(B-cell signaling and activation)等一系列免疫过程. 目前, 分离 和鉴定的唾液酸类化合物已达 50 余种, 其中 $N$-乙酰神 经氨酸(Neu5Ac, Sialic acid)、 $N$-差弪乙酰神经氨酸(Neu5-

*E-mail: ochemistry@163.com Tel.: +86 01062767947.

Received September 23, 2013; revised November 10, 2013; published online December 6, 2013.

Project supported by the Shenzhen Strategic Emerging Industrial Development Funds (No. JCYJ20130329174913512), and the China Postdoctoral Science Foundation (No. 2013M540013).

深圳市战略新兴产业发展专项资金(No. JCYJ20130329174913512)、中国博士后科学基金面上(No. 2013M540013)资助项目. 
$\mathrm{Gc}$ )和去氨基神经氨酸(KDN) 是最主要的 3 种存在形式. 由于 $N$-乙酰神经氨酸在自然界中分布最广, 因此又将 $N$-乙酰神经氨酸称为唾液酸.

唾液酸的最常见的生物合成途径如 Scheme 2 所示, UDP-GlcNAc 首先在 UDP-GlcNAc 2-epimerase 的催化 作用下转化为 $N$-乙酰甘露糖胺(ManNAc, 唾液酸的生 物合成前体), 然后 ManNAc 与 ATP 经 ManNAc kinase 催化生成 ManNAc-6-P (ManNAc-6-phosphate), ManNAc-6-P 再与 PEP (D-phosphoenolpyruvate) 经 Sialic acid-9-P synthase 催化生成 Sialic acid-9-P, 再经 Sialic acid-9-P phosphatase 去磷酸化生成唾液酸, 然后经 CMP-Sialic acid synthase 催化生成CMP-唾液酸, 最后由 糖基转移反应表达在细胞的糖链中.

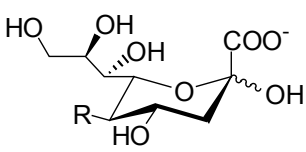

$R=N H A c($ Neu5Ac, Sialic acid)

$\mathrm{R}=\mathrm{NHGc}(\mathrm{Neu} 5 \mathrm{Gc}), \mathrm{R}=\mathrm{OH}(\mathrm{KDN})$

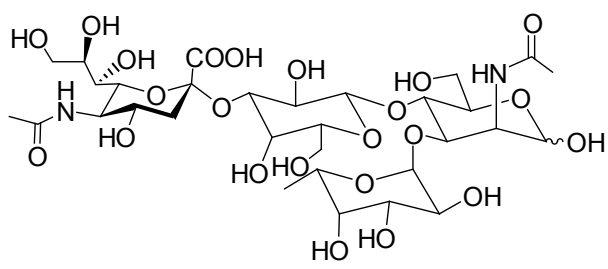

Sialyl LewX

\section{Scheme 1}<smiles>CCC(=O)N[C@H](O)[C@H](O)[C@H](O)COC(C)=O</smiles>

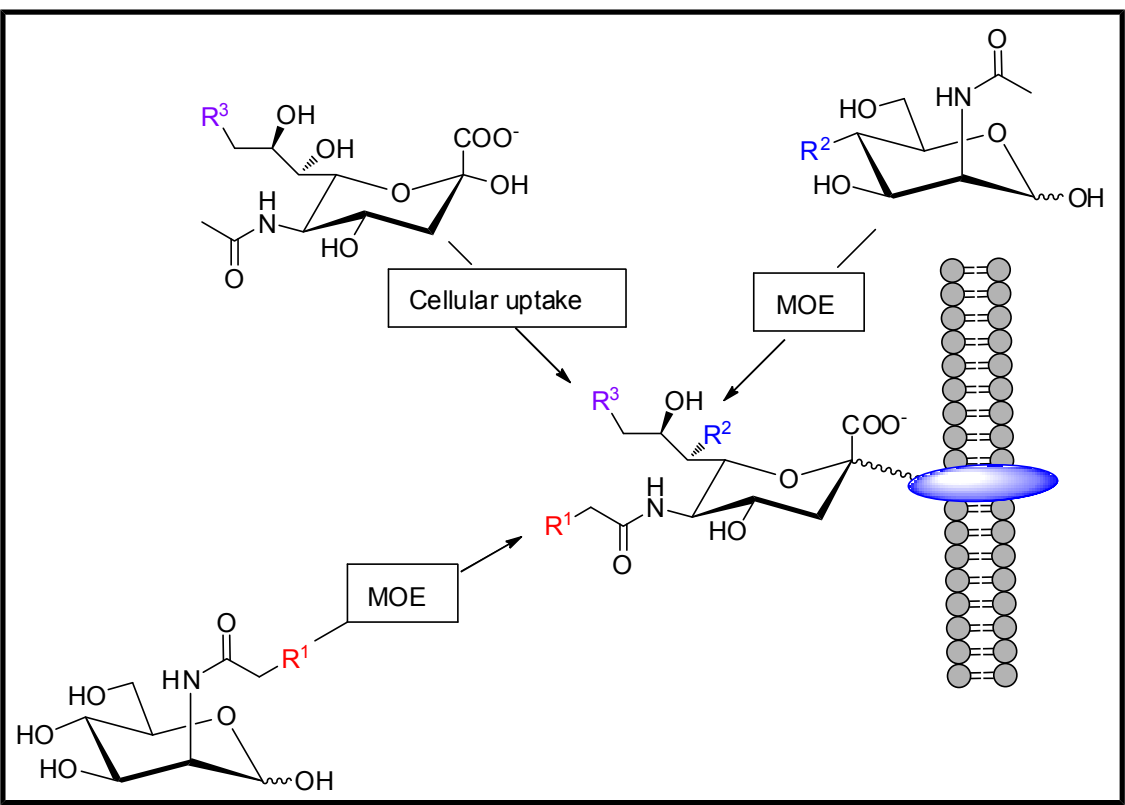

Scheme 2 

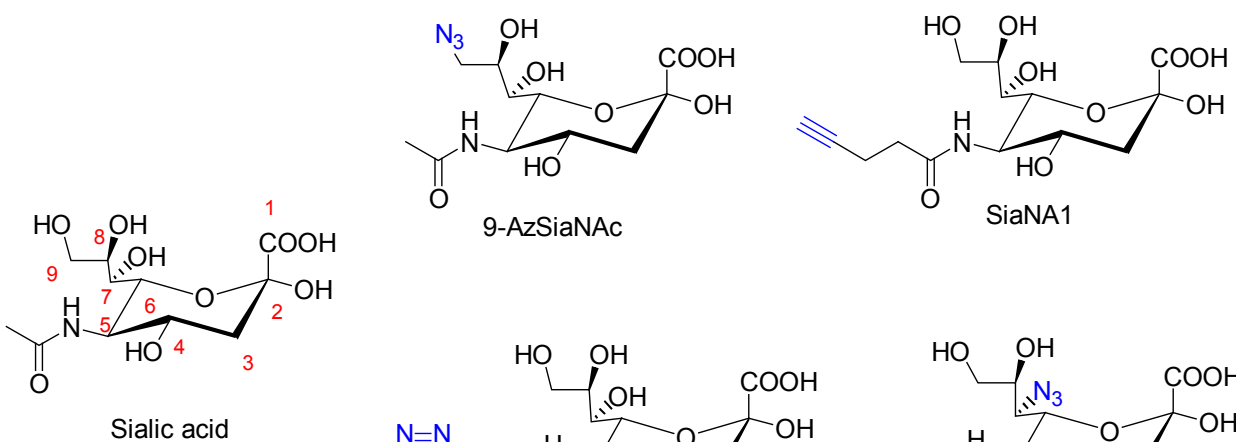

9-AzSiaNAc
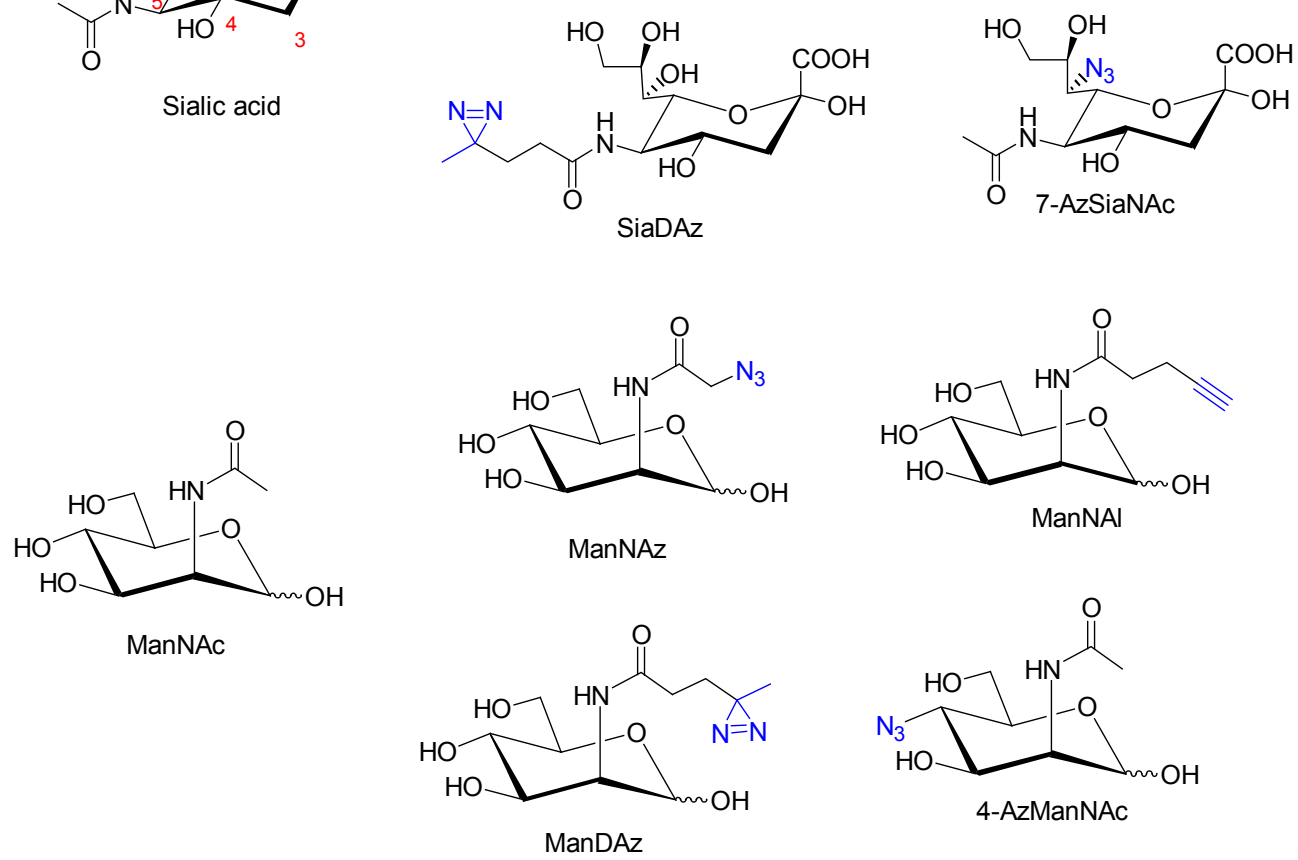

Scheme 3

由唾液酸的生物合成途径可知，除唾液酸的 2 号位 和 6 号位不能进行修饰外, 其他位点均可修饰, 而唾液 酸的生物合成前体 ManNAc 仅 $N$-乙酰位和 4 号位可进 行化学修饰. 经过化学家的孜孜努力, 采用甘露糖、葡 萄糖及其衍生物甚至非糖前体出发合成了大量的唾液 酸衍生物 ${ }^{[8]}$. 研究表明, 如果对唾液酸的 $N$-乙酰位、7 号位和 9 号位或 ManNAc 的 $N$-乙酰位和 4 号位进行化 学修饰, 可能不会影响其在细胞表面的表达 ${ }^{[9 \sim 12]}$. 基于 此, 研究人员对唾液酸的 $N$-乙酰位、7 号位和 9 号位和 其生物合成前体 ManNAc 的 $N$-乙酰位和 4 号位进行了 大量的化学修饰(Scheme 3), 所涉及的修饰基团主要包 括正交反应基团以及光交联基团等, 其中叠氮和端炔为 最重要的两个生物正交反应基团. 这两个基团可以进行 点击化学、Staudinger Ligation 等生物正交反应，从而偶 联上一系列探针. 而含有双呋丙啶(diazirine)和苯基叠 氮等光交联基团的非天然糖探针最近也被发展出来用 于捕获蛋白质与唾液酸之间的相互作用, 通过光交联高 通量的捕获与糖相互作用蛋白质, 对仪器设备要求不 高, 并且双呋丙啶和苯基叠氮是高效的光交联基团已用 于非天然糖代谢标记 ${ }^{[13,14]}$.

\section{1 号位的修饰}

细胞表面的唾液酸通常以负离子的形式存在, 其 1 号位的羧基阴离子与 $\mathrm{E}$-选择素键合而言至关重要 ${ }^{[15]}$. 此外, 这种阴离子间的电荷的排斥力可阻止红细胞互相 靠拢和聚集. Chan 等 ${ }^{[16,17]}$ 认为用磷酸基代替 1 号位的羧 基时，同样可提供负电荷，应该不影响唾液酸与 $\mathrm{E}$-选择 素的键合. 基于此，该课题组设计合成了唾液酸-1-磷酸 衍生物, 合成路线如 Scheme 4 所示. ManNAc 在铟的作 用下与 1 -溴甲基乙烯基磷酸二甲酯偶联得化合物 $\mathbf{1}$, 然 后直接将其氧化可得唾液酸-1-磷酸二甲酯(2). 化合物 1 在碱性条件下可水解为磷酸一甲酯 $\mathbf{3}$, 后者可氧化为唾 液酸-1-磷酸一甲酯 4 , 最后在 $\mathrm{TMSBr}$ 和碱的作用下脱 甲基得唾液酸-1-磷酸 5. 此外, TMSI 可直接水解掉化合 物 1 的两个甲基, 然后再氧化也可得唾液酸-1-磷酸 $5^{[18]}$.

Hagedorn 等 ${ }^{[19]}$ 采用 2-乙酰氨基-2-去氧呋喃甘露糖 衍生物 6 与 1,3 -丙酮二羊酸为起始原料, 在碱性条件及 醋酸镍的作用下缩合，再用二苯基重氮甲烷处理可得 1 位茮基保护的十碳糖 7, 后者在 $\mathrm{PdO}$ 的催化下氢化还原 脱保护得十碳糖 8 , 调 $\mathrm{pH}$ 至 $8 \sim 9$ 后再加热脱羒即可得 1 位修饰成甲基的唾液酸衍生物 $\mathbf{1 0}$ (Scheme 5). 
据 Zhang 等 ${ }^{[20]}$ 报道在三氟化硼乙醚的介导下五乙 酰唾液酸可与碳二亚胺类化合物缩合在 1,2 号位形成 $\alpha$ 型的螺环化合物, 其中 $R^{1}$ 和 $R^{2}$ 可为烷基、芳香基团等 (Eq. 1).

由于唾液酸 1 号位羧基的存在, 使其具有良好的水 溶性, 难以通过细胞质膜. 为解决以上问题, 可在酸性 条件下将唾液酸 1 号位羧基酯化以提高其脂溶性, 使唾 液酸更易渗透细胞膜进入细胞内. 唾液酸 1 号位的酯化 较为简单, 本文不再赘述.

\section{2 号位和 3 号位的修饰}

唾液酸 2 号位的羟基实质上是半缩酮羟基，其活性 反应活性往往高于其他差基，对 2 号位的修饰主要是成 醚或硫醚. 此类化合物经典的合成路线如下 ${ }^{[21 ~ 25]}$ : 首先 将唾液酸甲酯化(1-MeSiaNAc，12)和乙酰化(1-Me$\left.\mathrm{Ac}_{5} \mathrm{SiaNAc}, 13\right)$, 然后用氯取代 2 位的乙酰氧基，最后在 $\mathrm{AgOTf}$ 或 $\mathrm{AgBF}_{4}$ 的作用下与醇或硫醇缩合即可. 此合成<smiles>C=C(CBr)C(=O)OC</smiles>

3 $\mathrm{NaOH}$

(1) TMSI

(2) $\mathrm{O}_{3}$ then $\mathrm{Me}_{2} \mathrm{~S}$

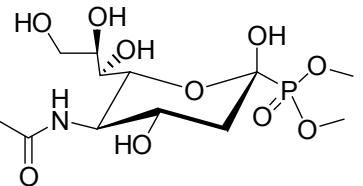

2

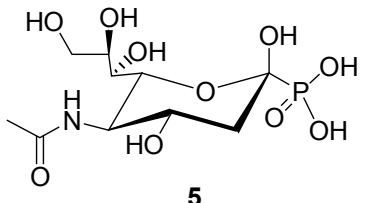

Scheme 4

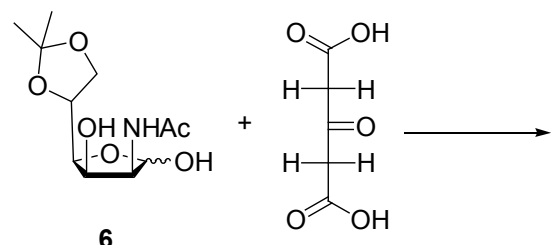<smiles>CC(=O)NC1[C@H](O)CC(O)(CC(=O)OCc2ccccc2)CC1(O)CO</smiles><smiles>CC(=O)NC1C(O)CC(O)(CC(=O)O)OC(O)C1[C@H](O)CO</smiles>

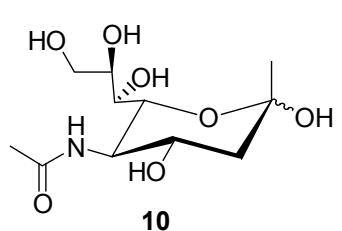<smiles>CC(=O)NC1C(O)CC(O)(CC(N)=O)CC(O)C1O</smiles>

Scheme 5

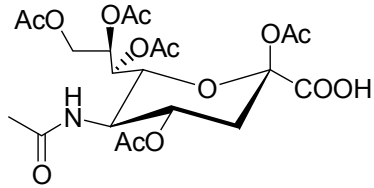

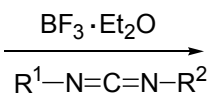

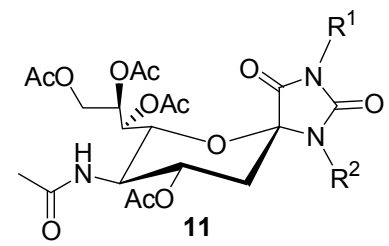


路线可进一步简化: 在乙酰氯的作用下 1-MeSiaNAc 可 直接转化为化合物 $\mathbf{1 4}^{[26,27]}$, 而 1-Me-Ac 5 SiaNAc 在三氟 化硼乙醚的催化下可直接与硫醇缩合 ${ }^{[28]}$. 另据报 道 ${ }^{[26,29 \sim 33]}$, 唾液酸的 2 号位为 SAc 时, 在碱性条件下可 发生取代反应将硫代酯 SAc 转化为硫醚 SR (Scheme 6).

最近, Noel 等 ${ }^{[34]}$ 报道了一种修饰唾液酸 2 号位的新 方法, 该方法以磷酸酯为原料在 TMSOTf 的作用下与亲 核试剂发生亲核取代反应，然后脱保护即可得 2 号位修 饰的唾液酸. 该方法具有良好的立体选择性, 对大部分
底物而言，仅生成 $\alpha$ 型产物(Scheme 7).

众所周知，在唾液酸的 3 号位引入氟原子可抑制唾 液酸的生物合成或影响唾液酸化 ${ }^{[35 \sim 42]}$, 故 3-氟唾液酸 常常作为唾液酸的抑制剂. 3-氟唾液酸可由唾液酸醛缩 酶 aldolase 催化相应的 3-氟丙酮酸钠与 ManNAc 缩合制 得 ${ }^{[42,43]}$, 也可由 $1-\mathrm{Me}-\mathrm{Ac}_{5} \mathrm{SiaNAc}$ 经脱水、氟化和乙酰化 制备 ${ }^{[44,45]}$. 其中，化合物 20 可进一步氟化得 2,3-二氟唾 液酸 $\mathbf{2 2}^{[43,46]}$ (Scheme 8).

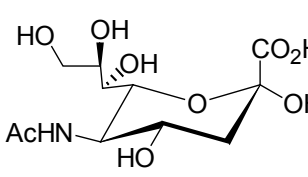

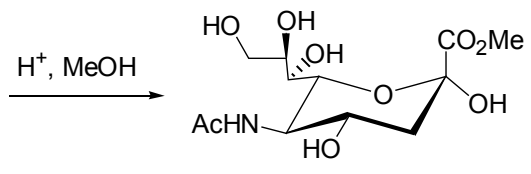

12
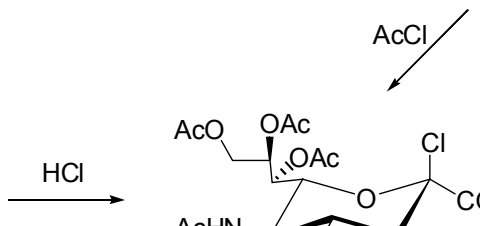

14

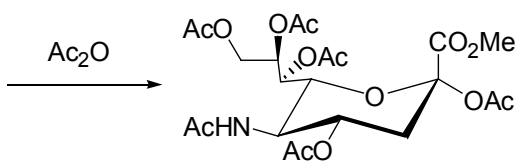

13

$\mathrm{RSH}, \mathrm{BF}_{3} \cdot \mathrm{Et}_{2} \mathrm{O}$ /or ROH, DIPEA<smiles>[X][R]O[Sb]</smiles>

15

Scheme 6

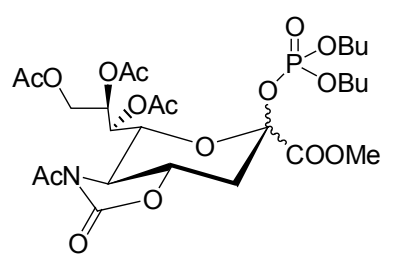

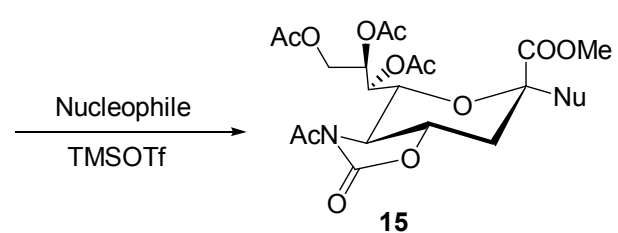

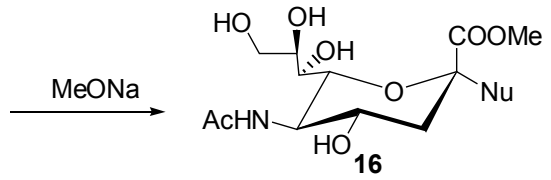

Scheme 7

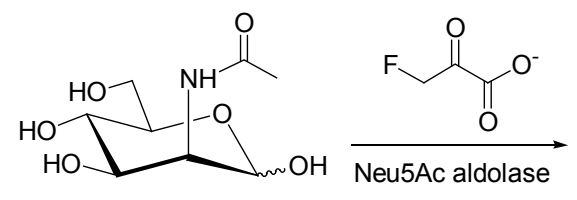<smiles>CC(=O)NC1C(O)[C@H](F)[C@@](O)(C(=O)[O-])OC1(O)C(=O)[O-]</smiles>

17

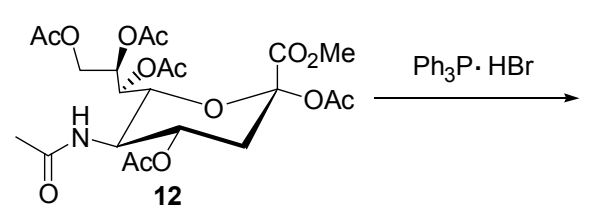

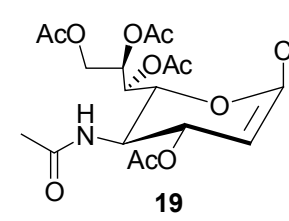<smiles>CO[N+](=O)[O-]</smiles>

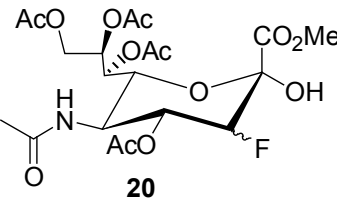<smiles></smiles>
DAST

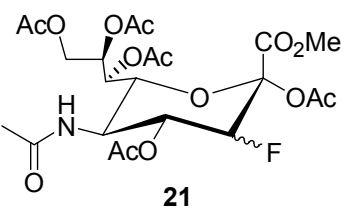

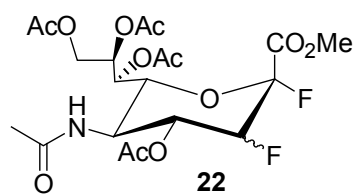

Scheme 8 
除氟化外, 化合物 19 可与 NBS 和水反应生成 3-溴 唾液酸衍生物 23, 后者在 $\mathrm{AIBN}$ 的作用下与 $\mathrm{Bu}_{3} \mathrm{SnAll}$ 发生烷基化反应可得立体专一性的 $3 \mathrm{e}$-烯丙基取代的唾 液酸 $\mathbf{2 4}^{[47,48]}$ (Scheme 9).

\section{4 号位的修饰}

Zanamivir 又称 Relenza, 其化学名为 $N$-乙酰基-2,3二去氧-4-胍基唾液酸(简称 4-胍基-神经氨-5-乙酰-2-烯), 是一个有效的流感病毒唾液酸抑制剂, 主要用于流感的 预防和治疗 ${ }^{[49]}$, 本品于 1999 年批准上市. 本品显著的 结构特点是在唾液酸的 4 号位引入胍基，同时 2,3 号位 失水成烯, 因此可将其看作是 4 位修饰的唾液酸衍生物. Zanamivir 及其衍生物可由下列路线合成: $1-\mathrm{Me}-\mathrm{Ac}_{5} \mathrm{SiaNAc}_{\mathrm{a}}$ 在 TMOSTf 的作用下转化为化合物 $\mathbf{2 5}$,
后者与 $\mathrm{TMSN}_{3}$ 发生 $\mathrm{S}_{\mathrm{N}} 2$ 取代反应在 4 号位引入叠氮, 然 后将叠氮还原为氨基可得关键中间体 27 , 最后通过脱 保护与氨基上引入取代基等反应即可得 Zanamivir 及其 衍生物 $\mathbf{2 8}^{[50,51]}$ (Scheme 10).

将化合物 19 脱乙酰保护, 然后选择性的保护 8,9 号 位得中间体 30, 后者 4 号位的羟基在强碱的作用下可与 卤代烷反应成醚，最后脱 8,9 号位和 1 号位的保护可得 4 号位成醚的唾液酸衍生物 $\mathbf{3 3}^{[51 ~ 53]}$ (Scheme 11).

$\mathrm{Ye}$ 等 ${ }^{[54}$ 56]报道了一种在唾液酸的 4 号位直接引入 哌嗪衍生物的新方法, 该法以化合物 19 为原料, 在吡啶 的存在下与哌嗪衍生物缩合同时脱 2 号位乙酰基即可得 4 号位修饰的唾液酸衍生物 34. 其中, $\mathrm{R}$ 可含有叠氮、炔 基等生物正交反应基团，这样就可以在唾液酸的 4 号位 进行生物正交反应，以拓展对唾液酸的研究(Eq. 2).

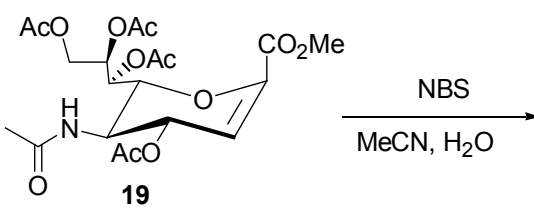

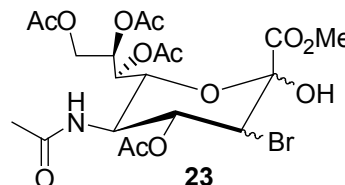

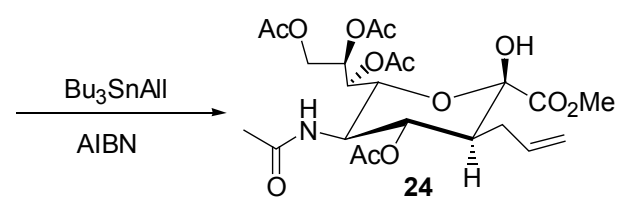

Scheme 9

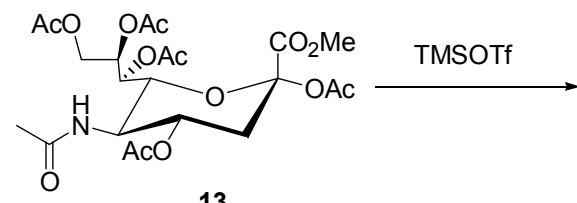

13

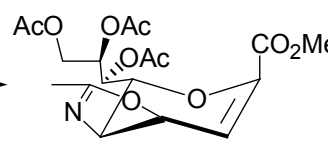

25

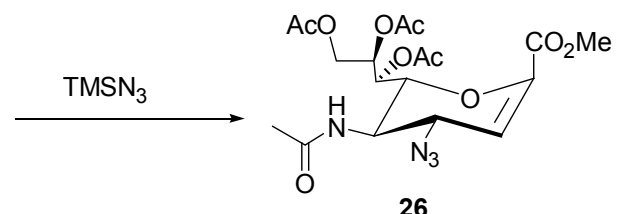

26

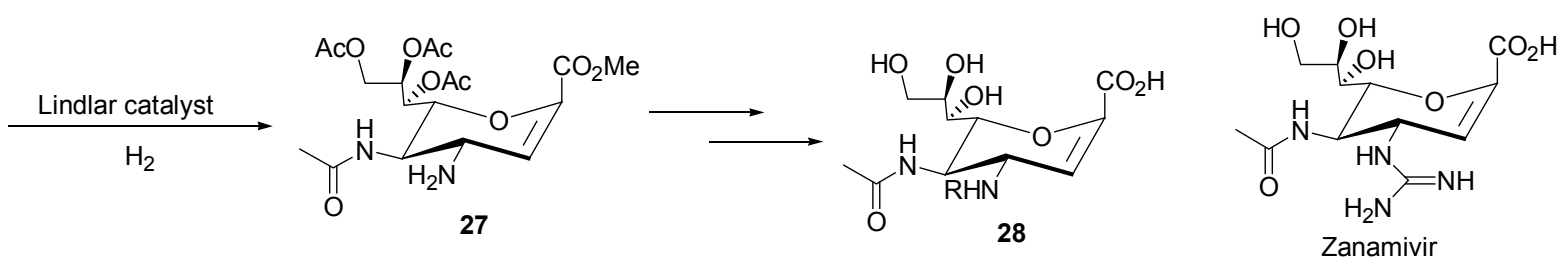

Scheme 10

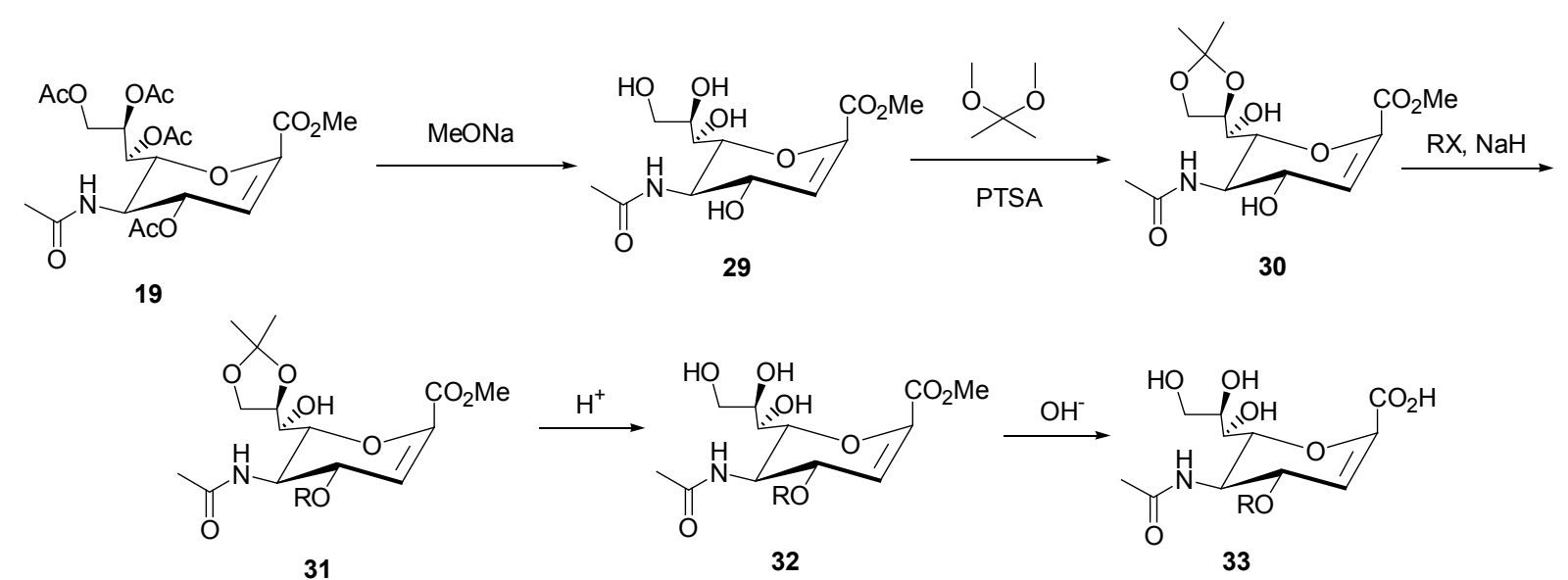

Scheme 11 


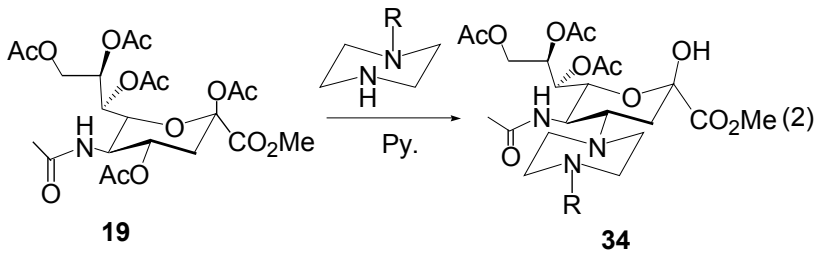

据文献 $[50,57 \sim 59$ ]报道, $D$-葡萄糖- $\delta$-内酯可通过 保护、还原、取代、氧化等 8 步反应转化为关键中间体 35, 然后通过 1,3-偶极环加成等反应成五元杂环 36, 后 者在丙烯酸甲酯的作用下转化为化合物 $\mathbf{3 7}$, 再经氢化、 保护、氧化、脱保护、中和等步骤得到了极为重要的 4氨基唾液酸 40, 该化合物的氨基可进一步修饰制备一 系列 4 号位含有取代胺基的唾液酸衍生物(Scheme 12).

\section{$4 \mathrm{~N}$-乙酰位的修饰}

唾液酸可在唾液酸醛缩酶的催化作用下降解为 ManNAc 和丙酮酸 ${ }^{[60]}$, 人们马上意识到可利用这一降解 的逆过程来合成唾液酸. 事实上, 目前绝大多数 $N$-乙酰 位的非天然唾液酸是由唾液酸醛缩酶 aldolase 催化相应 的 ManNAc 衍生物与丙酮酸反应制得 ${ }^{[14,61 ~ 65]}$. 研究表 明, 取代基的电子效应和疏水作用影响 aldolase 的催化
效率，如当 $\mathrm{R}$ 为强吸电子基时 aldolase 的催化效率将大 幅下降 ${ }^{[61]}$ (Scheme 13).

研究表明 ${ }^{[66,67]}$, 当甘露糖胺的氨基用 Boc 或 $\mathrm{Cbz}$ 等 保护基保护时, aldolase 可将其转化为相应的唾液酸衍 生物，此时脱保护可得含有游离氨基的关键中间体，然 后将 5 位的氨基与羧酸的衍生物进行缩合即可得目标 物. 此外, 2-叠氮甘露糖可经 aldolase 转化为 5-叠氮唾 液酸, 将叠氮还原后也可得关键中间体 5-氨基唾液酸 $\mathbf{4 3}^{[68]}$ (Scheme 14).

如上所述, $1-\mathrm{MeAc}_{5} \mathrm{SiaNAc}$ 的 2 位酯基可转化为醚 或者硫醚 44, 2 位修饰的唾液酸衍生物再经脱乙酰基保 护可得 5 位含有游离氨基的唾液酸衍生物 46, 后者再与 羧酸的衍生物进行缩合，最后经乙酰基保护羟基、脱 2 位醚或者硫醚保护和脱乙酰保护等过程可得 $N$-乙酰位 修饰的唾液酸 $\mathbf{5 0}^{[69 \sim 71]}$. 此外, 唾液酸在酸催化下可直 接与甲醇作用同时保护 1 位(形成甲酯)和 2 位(形成甲 醚) ${ }^{[72]}$, 而当 Dowex-50 $\left(\mathrm{H}^{+}\right)$换成 $\mathrm{HCl}$ 时, 不仅可保护 1 位(形成甲酯)和 2 位(形成甲醚), 而且还可同时脱掉 5 位 的乙酰基得化合物 46, 使合成路线进一步简化 ${ }^{[73]}$ (Scheme 15).<smiles>CC#CC(=O)C(N)C1OC(C)(C)OC1C1CN(C)C(C)(C)O1</smiles>

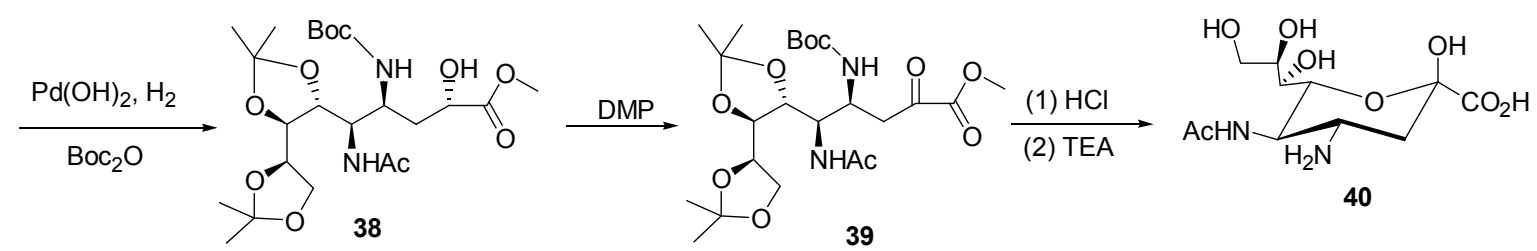

Scheme 12

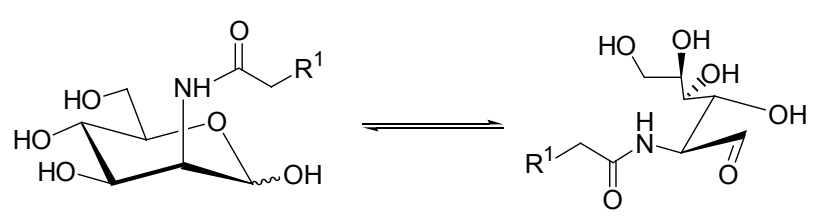

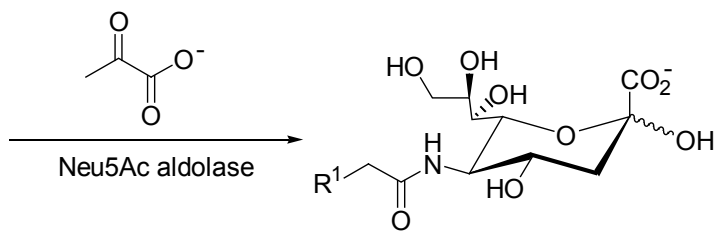

Scheme 13 


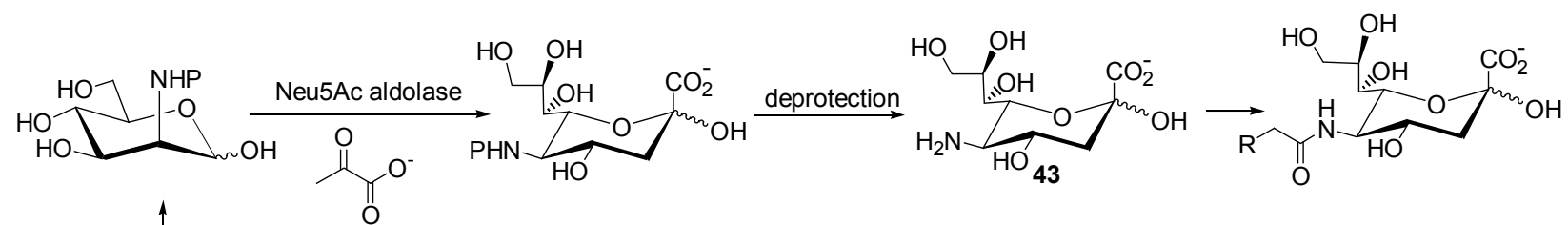

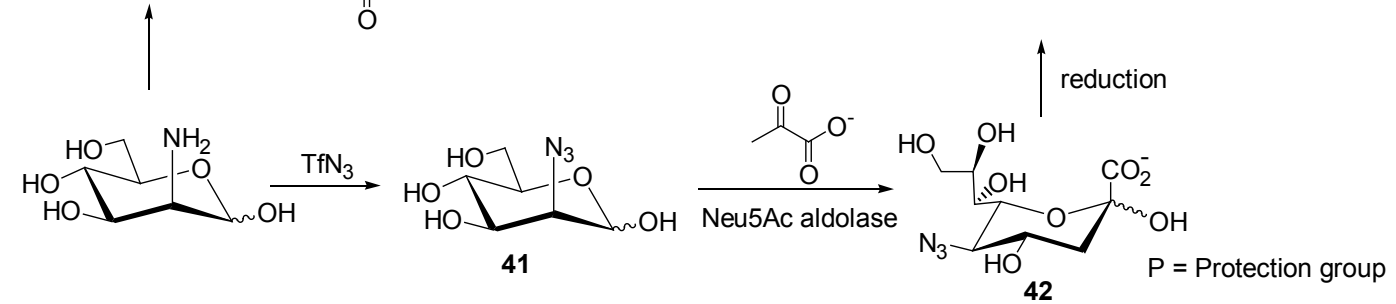

\section{Scheme 14}

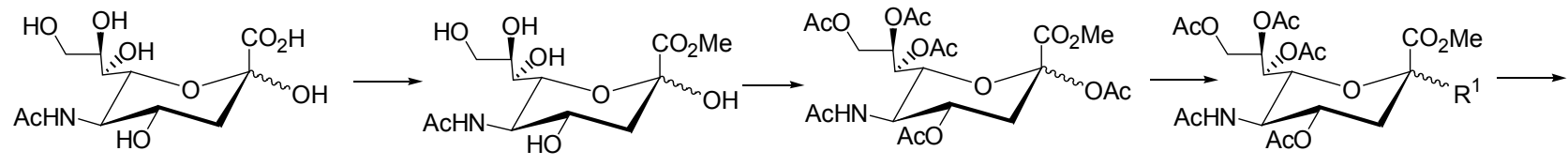

12

13

44

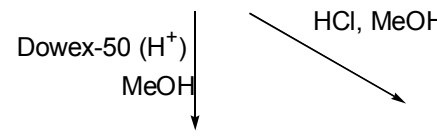

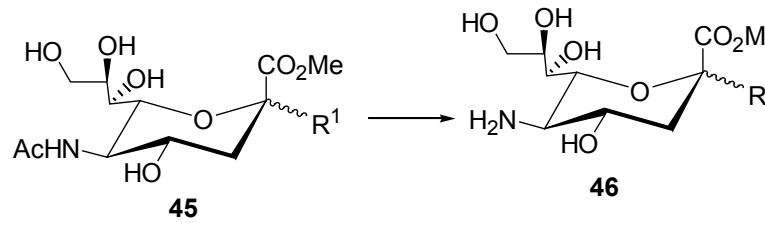

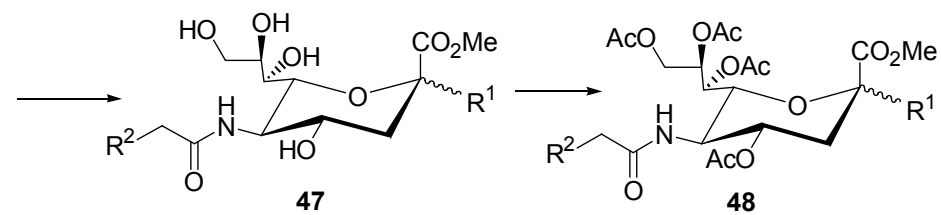

(1)

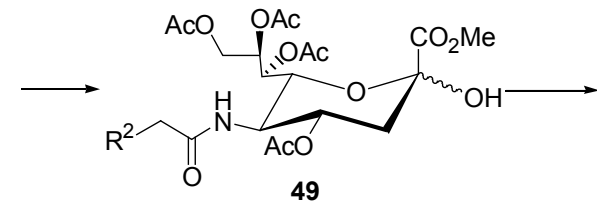

49

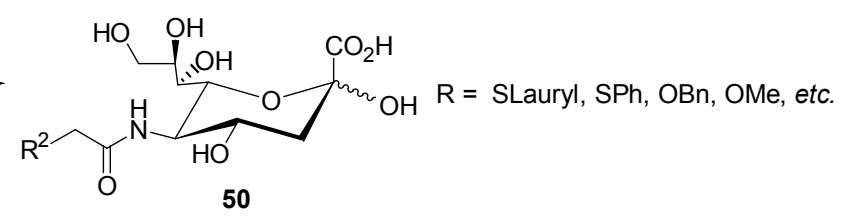

Scheme 15

\section{7 号位的修饰}

唾液酸的 7 号位很难直接进行修饰, 一般是由 ManNAc 首先合成 4-取代- $N$-乙酰基甘露糖胺衍生物, 然后经 aldolase 催化与丙酮酸钠反应制得. 具体合成路 线如下: 首先将 ManNAc 的 6 号位 Ts 化, 接着在 DBU 的作用下 1,6 位形成内醚 52, 后者发生 Mitsunobu 反应 得 3,4 位环化产物 $\mathbf{5 3}$, 然后化合物 $\mathbf{5 3}$ 与亲核试剂发生 $\mathrm{S}_{\mathrm{N}} 2$ 反应将 3,4 位的环氧丙烷结构打开同时在 4 号位引 入取代基, 再在 TMSOTf 和乙酸䣶的作用下打开 1,6 位 所形成的内醚同时乙酰化, 然后脱乙酰基得 4-取代- $N$ 乙酰基甘露糖胺衍生物 56, 最后将 4-取代- $N$-乙酰基甘 露糖胺衍生物在 aldolase 催化下与丙酮酸钠缩合即可得 7 号位修饰的唾液酸衍生物 ${ }^{[12,74 ~ 76]}$ (Scheme 16).

\section{8 号位的修饰}

众所周知, 唾液酸以 $\alpha-2,8$-糖苷键连接所形成的多 聚唾液酸(PSA)是一些哺乳动物细胞中糖蛋白的组成部 分, 而且对脑部发育、调节神经细胞黏附分子及其他黏 附分子介导的细胞间相互作用等生物过程具有重要作 用 ${ }^{[77 ~ 82]}$. 因此, 有必要对唾液酸的 8 号位进行修饰以进 一步研究其所参与的生物过程.

对唾液酸 8 号位的修饰主要有两种方法: (1)以唾液 酸为起始原料, 将 1,2 位进行保护, 接着保护 8,9 位和 4 号位，选择性的脱 8 位保护，然后在三氟甲磺酸䣲的作 用下 7,8 位成环氧丙烷结构, 脱 9 号位保护后用亲核试 剂进攻 8 号位，最后脱保护可得 8 号位修饰的唾液酸衍 生物 ${ }^{[82 ~ 84]}$ (Scheme 17); (2)以廉价易得的 1,2:5,6-二异 

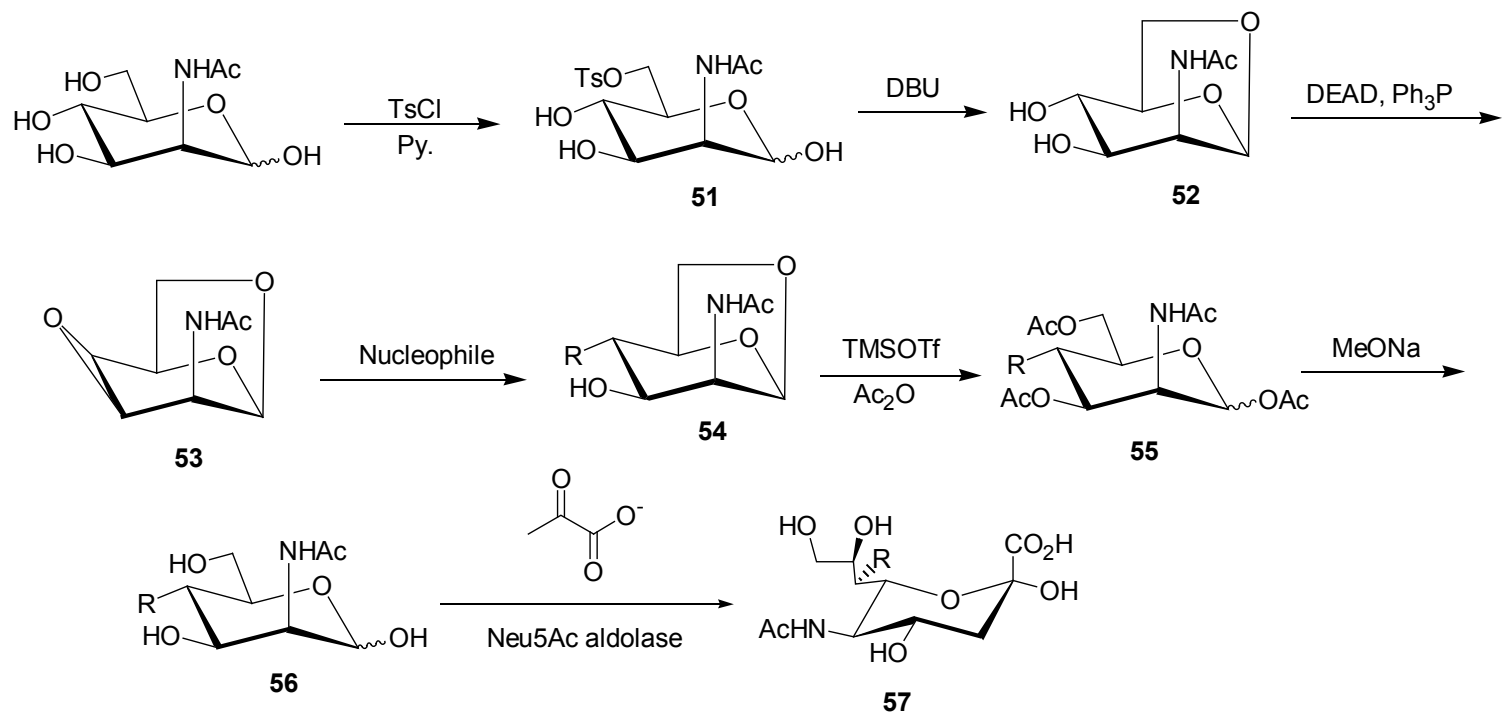

Scheme 16

亚丙基- $\alpha-D$-吡喃葡萄糖 65 为起始原料，经保护、取代、碳糖 68, 后者在 PmNanA 酶的作用下与丙酮酸钠缩合 选择性脱保护等 5 步反应得中间体 66, 然后用卤代烷与 即得 8 号位修饰有醚键的唾液酸衍生物 ${ }^{[85}$ (Scheme 18).

差基成醚得化合物 67, 再经还原、乙酰化和脱保护得六
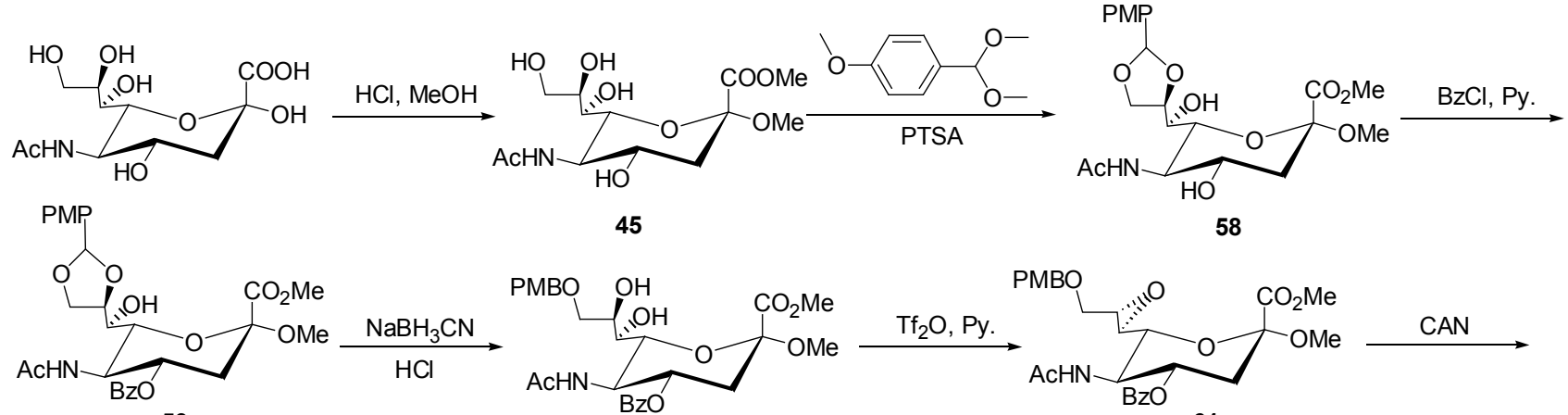

45

58

60

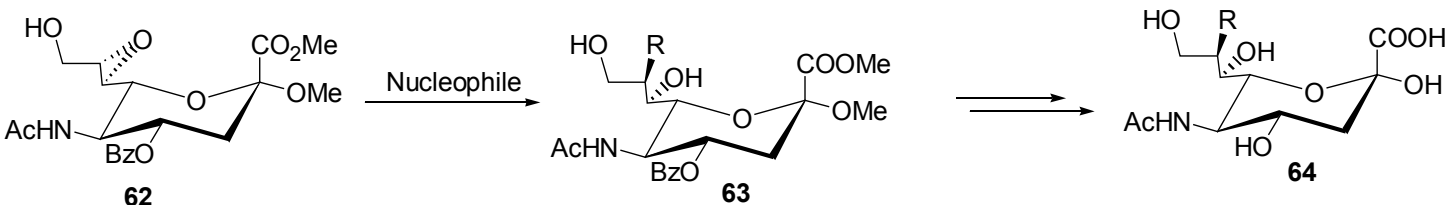

Scheme 17

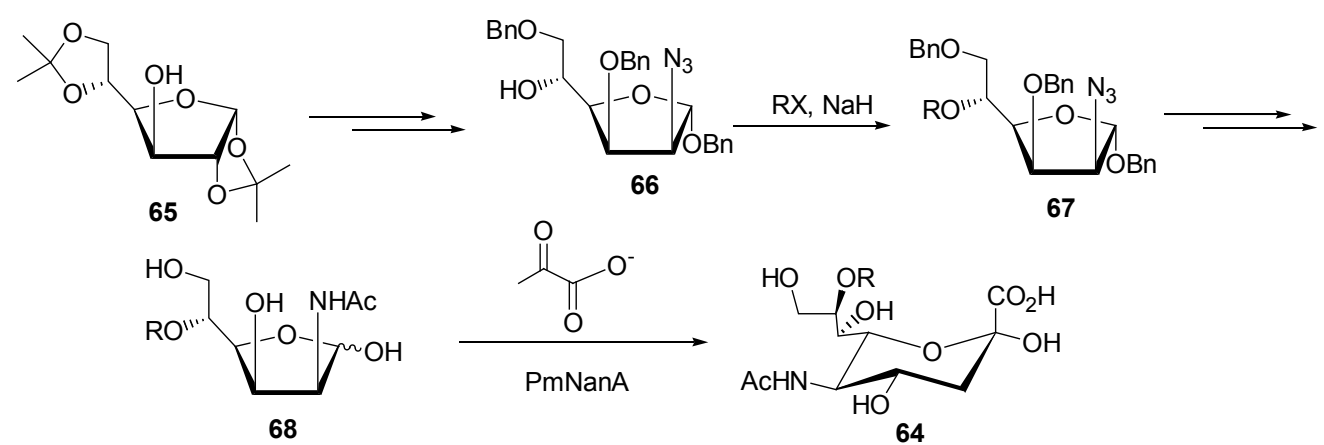

Scheme 18 


\section{9 号位的修饰}

唾液酸的 9 号位对细胞一细胞间的相互作用、细胞 分化、肿瘤转移和致病菌识别宿主等生物过程极为重

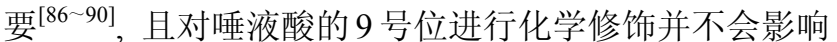
其整合到细胞表面糖蛋白上，因此，近年来研究人 员 ${ }^{[91 ~ 100]}$ 对唾液酸的 9 号位进行了大量的修饰.

其中, 在唾液酸的 9 号位引入叠氮或将其还原成氨 后再引入其他修饰基团如生物正交反应基团(如叠氮和 炔基)和光交联基团(如双呋丙啶和对叠氮苯甲酰基)研 究的最为广泛. 此类化合物一般有两种合成方法: (1)以 1-MeSiaNAc 为起始原料, 经对 9 号位的 Ts 化和叠氮化 可得 9-AzSiaNAc 70, 然后将叠氮氢化还原成氨, 最后 与羧酸缩合可得目标物 $\mathbf{7 2}^{[91 ~ 98]}$; (2) 以化合物 51 为起始 原料, 经叠氮化转化为 6-AzManNAc 73, 后者在 aldolase 的催化下与丙酮酸钠反应转化为 9-AzSiaNAc,
最后经还原、缩合等反应也可得 9 位修饰的唾液酸衍生 物 $\mathbf{7 2}^{[99]}$ (Scheme 19).

Kiefel 等 ${ }^{[90]}$ 设计合成了 9 位为羧基的唾液酸衍生物 76, 合成路线如下: 首先用 TBDMS 保护化合物 45 的 9 号位，然后用乙酰基保护其他羟基，再在酸性条件下脱 9 号位保护得化合物 74, 后者经氧化、脱保护等步骤可 得 9 位为着基的唾液酸衍生物 76. 研究表明，化合物 45 经氧化和乙酰化两步可直接制备化合物 75, 使合成路 线进一步简化(Scheme 20).

Martin 等 ${ }^{[100]}$ 以 $1-\mathrm{MeSiaNAc}$ 为原料经过与 $\mathrm{MePO}(\mathrm{OH})_{2}$ 酯化、脱保护等反应得到了 9 号位为亚磷酸 酯的唾液酸衍生物 78 (Scheme 21).

以 ManNAc 为起始原料, 将其 6 号位酯化, 然后在 aldolase 的催化下与丙酮酸钠反应也可转化为 9 号位修 饰有酯基的唾液酸衍生物 ${ }^{[101]}$ (Scheme 22).

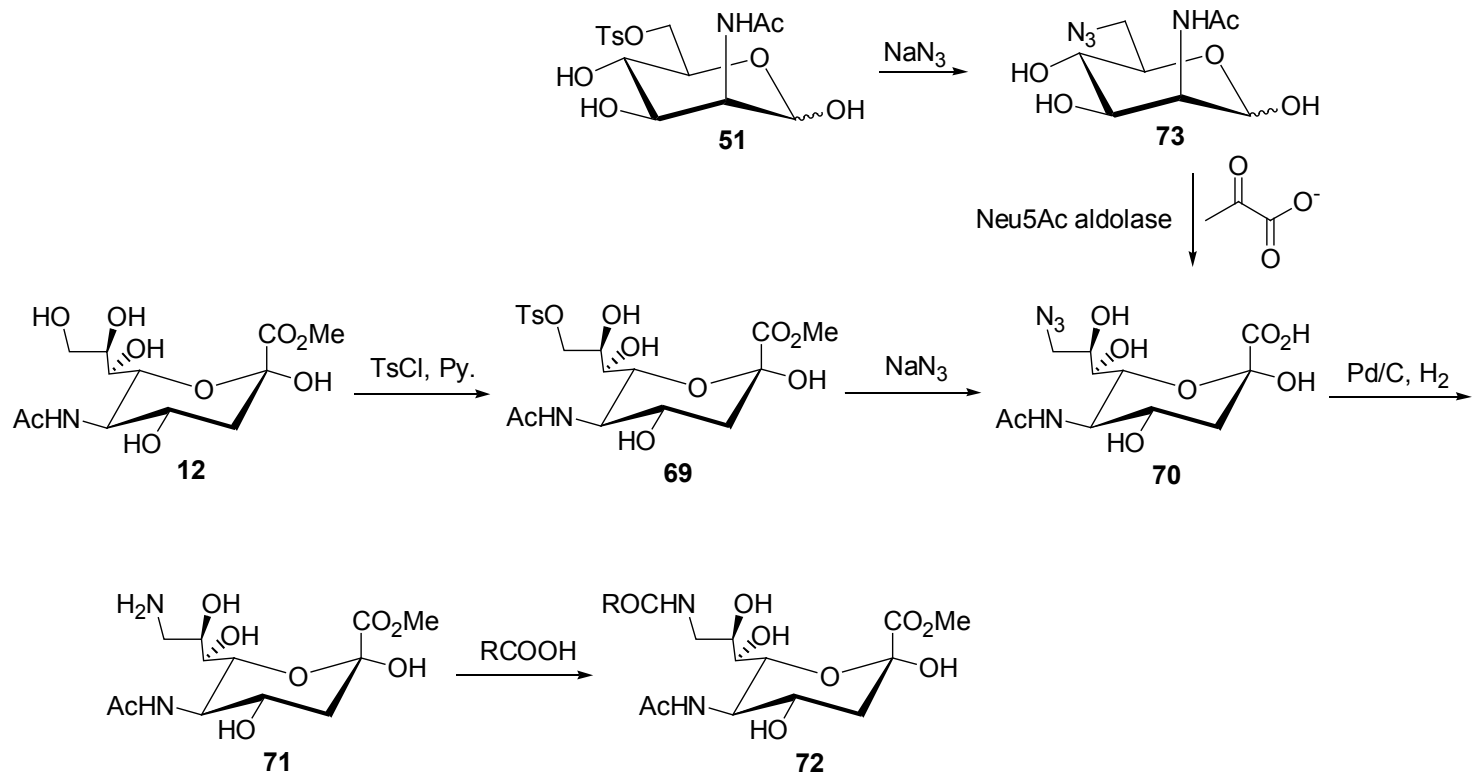

Scheme 19

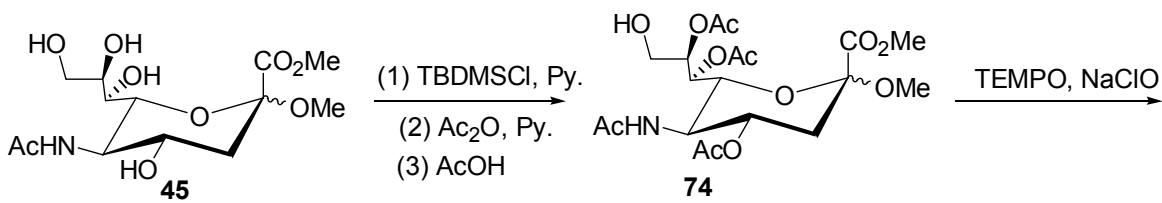

TEMPO, $\mathrm{NaClO}$ $\checkmark \mathrm{Ac}_{2} \mathrm{O}, \mathrm{Py}$
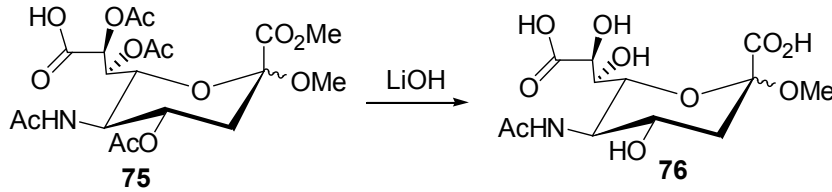

Scheme 20 


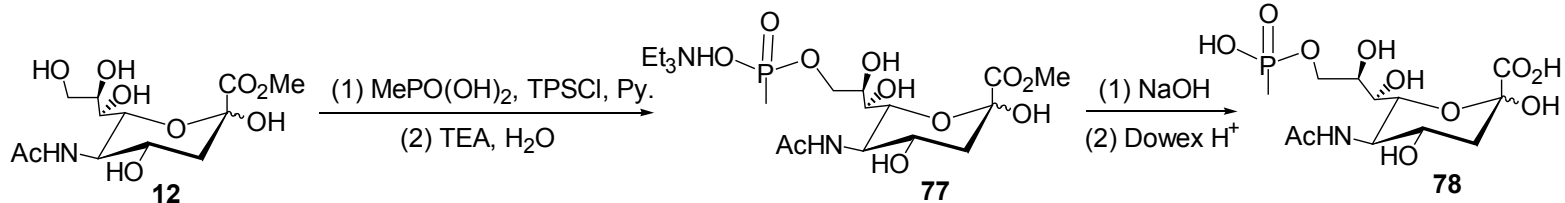

Scheme 21

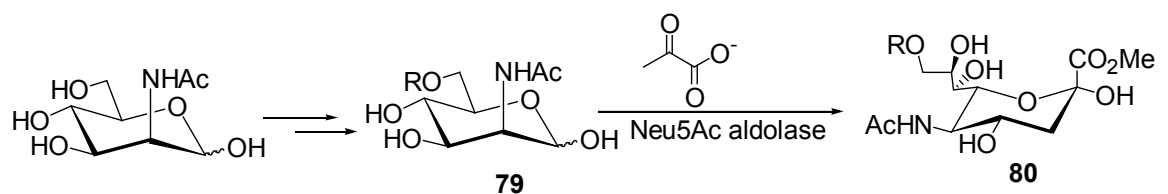

Scheme 22

\section{8 其他}

迄今为止, 所有发展出来的非天然糖探针均只含有 一个非天然官能团即均为非天然单功能糖, 这就极大地 限制了非天然糖在研究糖与蛋白质动态相互作用中的 应用。

基于在唾液酸 $N$-乙酰位和 9 号位上均有成功修饰 的非天然糖探针, 本课题组 ${ }^{[102]}$ 在 $N$-乙酰位和 9 号位同 时引入生物正交反应基团(叠氮、炔基等)和光交联基团 (双叫丙啶等), 设计合成两个非天然双功能糖探针即 9-AzSiaNAl 和 9-AzSiaDAz，合成路线如 Scheme 23.

其中, 9-AzSiaNAl 经代谢过程表达到细胞表面后, 我们可以采用两种互相不干扰的生物正交反应, 分别接
上两种探针. 两种生物正交反应的选择可以为：第一步 用张力诱导环加成反应(strain-promoted cycloaddition, 又被称为 copper free click chemistry)通过 $N$-乙酰位的叠 氮与探针 A 相连接; 第二步使用 BTTES 协助的点击化 学(BTTES-assisted CuAAC)在 9 号位上的端炔上与探针 $\mathrm{B}$ 相连. 最近发展的 BTTES-assisted CuAAC 反应解决 了传统点击化学铜生物毒性问题, 使其可应用于活细 胞. 这一方法在两种探针的选择上具有多种组合的可 能, 如 $\mathrm{C}$ 和 $\mathrm{D}$ 可以是两种不同颜色的荧光分子, 用于双 色成像; $\mathrm{C}$ 和 $\mathrm{D}$ 可以是一对产生荧光能量共振转移 (Forster Resonence Energy Transfer, FRET)的苂光分子, 即一个为 doner, 另一为 acceptor. FRET 可以大大降低聚<smiles>C#CCCC(=O)OCCCCCCCCCC(=O)ON1C(=O)CCC1=O</smiles><smiles>[R]CC(=O)NC1(CO)C(O)C(O)C(O)C1[C@H](O)CC</smiles><smiles>[R]CC(=O)NC1C(O)CC(O)(C(=O)[O-])CC(O)C1[C@H](O)CO</smiles><smiles>[R]CC(=O)NC1C(O)CC(O)(C(C)=O)OC(O)C1C(O)CO</smiles>

89

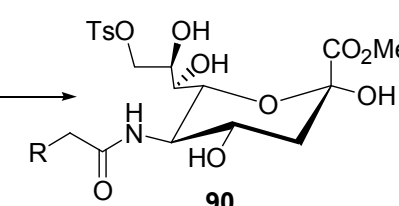

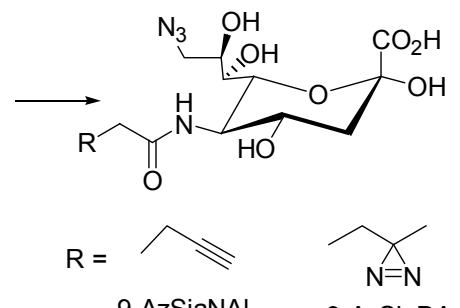

Scheme 23 
糖苂光分子成像的背景, 更重要的是可以进行聚糖的动 态成像与观测.

而 9-AzSiaDAz 可通过 $365 \mathrm{~nm} \mathrm{UV}$ 光照, 实现对细 胞内和细胞膜上与唾液酸相互作用的蛋白质的光交联; 然后应用唾液酸苷酶从唾液酸上切掉细胞裂解液中所 有被唾液酸修饰的蛋白质; 接着通过生物正交反应，如 铜离子催化的叠氮一炔基环加成反应(CuAAC reaction) 使非天然唾液酸上叠氮与带有炔基的生物素(Biotin)反 应，生物素则与亲和素 Beads 结合，实现对与糖相互作 用蛋白质的捕捉; 然后通过胶内酶切和液相色谱-二级 质谱鉴定捕捉的蛋白. 最后, 结合文献与生物信息手段 分析结果, 选出结果可靠性高并具有重大生物学意义的 蛋白质, 结合传统生物化学与分子生物学方法深入研究 其与糖的相互作用对细胞功能的影响. 通过光交联高通 量的捕获与糖相互作用蛋白质, 对仪器设备要求不高, 并且双呋丙啶和苯基叠氮是高效的光交联基团, 并已经 被证明可以用于非天然糖代谢标记. 而生物正交反应基 才可以作为蛋白富集的标签，其相比于蛋白融合标签， 具有尺寸小、特异性高等优势. 更为重要的是, 这个基 团还可以连接重金属, 因此可以结合电镜技术, 实现对 糖基化修饰蛋白在亚细胞水平上的高分辨观察.

\section{9 展望}

尽管聚糖在各种基本生物过程和相关人类疾病研 究中的重要性已经得到广泛的关注, 然而, 相对于对蛋 白质和核酸等生物大分子的研究, 科学家们在对聚糖在 分子层面的功能解析是相对滞后的 ${ }^{[103]}$. 造成这个局面 最重要原因之一是聚糖和其他生物大分子(例如核酸、 蛋白质等)不同，其生物合成不受基因模板控制. 这个 特性导致聚糖，特别是修饰在蛋白质上的聚糖通常是多 种多样、非均一的. 这种微观非均一性使其很难用传统 的基因技术来研究. 尽管对于体外生化研究来说，均一 的聚糖样品可以通过化学和酶学合成得到 ${ }^{[6]}$, 但是, 细 胞层面的研究要求能够在活体生理条件下对糖基化进 行操控和检测. 另一方面, 分子成像作为在活体细胞条 件下研究生物分子功能的有力工具, 已经被广泛用于蛋 白质 ${ }^{[104]}$ 、核酸 ${ }^{[105]}$ 等的研究中. 然而, 由于标记蛋白的 方法(如基于编码的荧光蛋白)和标记核酸的方法都无法 直接应用到聚糖的标记, 聚糖的分子成像手段亟待发 展. 化学糖生物学的研究主要是发展和应用化学工具研 究糖生物学, 其中, 通过化学手段在活体细胞上研究糖 基化的功能是这方面的热点. “非天然糖代谢工程” (Metabolic Oligosaccharide Engineering, MOE)的提出和 发展, 为解决在活体生理条件下对糖基化进行操控和检 测这一难题提供了可能性 ${ }^{[106]}$. Bertozzi 研究组 ${ }^{[63]}$ 是非天
然糖代谢工程技术研究的先驱者之一. 该课题组 ${ }^{[107]}$ 发 展了含有炔基的非天然糖探针用于细胞活体标记. 最 近，该组还把这项技术运用到了斑马鱼发育过程的研究 中. 该方法也很快的得到了糖生物学家的广泛关注, 并 且已被用于研究特定的糖生物学问题.

唾液酸具有重要的生物学功能，在抗炎、抗肿瘤、 抗病毒、抗老年痴呆等领域发挥着极为重要的作用，引 起了各国学者的广泛关注. 唾液酸可修饰的位点较多, 目前已发展出成千上万的非天然唾液酸衍生物用于分 子成像。尽管如此，非天然糖代谢工程方法还处于发展 的初期，很多方面亟待发展和完善.

\section{References}

[1] Somers, W. S.; Tang, J. Cell 2000, 103, 467.

[2] Rosen, S. D. Annu. Rev. Immunol. 2004, 22, 129.

[3] Lopez, P. H. H.; Schnaar, R. L. Curr. Opin Struct. Biol. 2009, 19, 549.

[4] Dube, D. H.; Bertozzi, C. R. Nat. Rev. Drug Discovery 2005, 4, 477.

[5] Chen, X.; Varki, A. ACS Chem. Biol. 2010, 5, 163.

[6] Bertozzi, C. R.; Kiessling L. L. Science 2001, 291, 2357.

[7] Cheng, C.; Gao, C.-F. Lab. Med. 2013, 4, 333 (in Chinese). (程铖, 高春芳, 检验医学, 2013, 4, 333.)

[8] Li, L.-S.; Liu, K.-G.; Yao, Z.-J.; Wu, Y.-L. Chin. J. Org. Chem. 2002, 22, 718 (in Chinese).

(李连生, 刘克刚, 姚祝军, 吴毓林, 有机化学, 2002, 22, 718.)

[9] Luchansky, S. J.; Goon, S.; Bertozzi, C. R. ChemBioChem 2004, 5(3), 371.

[10] Oetke, C.; Brossmer, R.; Mantey, L. R.; Hinderlich, S.; Isecke, R.; Reutter, W.; Keppler, O. T.; Pawlita, M. J. Biol. Chem. 2002, 277(8), 6688.

[11] Oetke, C.; Hinderlich, S.; Brossmer, R.; Reutter, W.; Pawlita, M.; Keppler, O. T. Eur. J. Biochem. 2001, $268(16), 4553$.

[12] Möller, H.; Böhrsch, V.; Bentrop, J.; Bender, J.; Hinderlich, S.; Hackenberger, C. P. R. Angew. Chem., Int. Ed. 2012, 51, 5986.

[13] Han, S.; Collins, B. E.; Bengtson, P.; Paulson, J. C. Nat. Chem. Biol. 2005, 1(2), 93.

[14] Tanaka, Y.; Kohler, J. J. J. Am. Chem. Soc. 2008, 130, 3278.

[15] Uchiyama, T.; Vassilev, V. P.; Kajimoto, T.; Wo, C.-H. J. Am. Chem. Soc. 1995, 117, 5395.

[16] Chan, T.-H.; Xin, Y.-C. Chem. Commun. 1996, 1(8), 905.

[17] Chan T.-H., Xin, Y.-C. J. Org. Chem. 1997, 62, 3500

[18] Gao, J.; Martichonok, V.; Whitesides, G. M. J. Org. Chem. 1996, 61,9538 .

[19] Hagedorn, H.; Merten, H.; Brossmer, R. Carbohydr. Res. 1992 236,89 .

[20] Zhang, D.-Y.; Ye, D.-J.; Wang, J.-F.; Shi, J.-M.; Jiang, H.-L.; Liu, H. J. Org. Chem. 2010, 75, 3552.

[21] Pan, Y.; Ayani, T.; Nadas, J.; Wen, S.; Guo, Z. Carbohydr. Res. 2004, 339(12), 2091.

[22] Ikeuchi, Y.; Sumiya, M.; Kawamoto, T.; Akimoto, N.; Mikata, Y.; Kishigami, M.; Yano, S.; Sasakic, T.; Yoneda, F. Bioorg. Med. Chem. 2000, 8, 2027.

[23] Knoll, T. L.; Bennet, A. J. J. Phys. Org. Chem. 2004, 17, 478.

[24] Chou, D. T. H.; Watson, J. N.; Scholte, A. A.; Borgford, T. J.; Bennet, A. J. J. Am. Chem. Soc. 2000, 122, 8357.

[25] Zamora, C. Y.; Alarcao, M.; Kumar, K. Bioorg. Med. Chem. Lett. 
2013, 23, 3406 .

[26] Sakamoto, J. I.; Koyama, T.; Miyamoto, D.; Yingsakmongkon, S.; Hidari, K. I. P. J.; Jampangern, W.; Suzuki, T.; Suzuki, Y.; Esumi, Y.; Nakamura, T.; Hatano, K.; Terunuma, D.; Matsuoka, K. Med. Chem. 2009, 17, 5451 .

[27] Hinou, H.; Kurogochi, M.; Shimizu, H.; Nishimura, S. Biochem. 2005, 44, 11669.

[28] Matsuoka, K.; Onaga, T.; Mori, T.; Sakamoto, J. I.; Koyama, T.; Sakairi, N.; Hatano, K.; Terunuma, D. Tetrahedron Lett. 2004, 45, 9383.

[29] Abo, S.; Ciccotosto, S.; Alafaci, A.; Itzstein, M. Carbohydr. Res. 1999, 322, 201.

[30] Matsuoka, K.; Takita, C.; Koyama, T.; Miyamoto, D.; Yingsakmongkon, S.; Hidari K. I. P. J.; Jampangern, W.; Suzuki, T.; Suzuki, Y.; Hatano, K.; Terunuma, D. Bioorg. Med. Chem. Lett. 2007, 17, 3826.

[31] Morais, G. R.; Oliveira, I. F.; Humphrey, A. J.; Falconer, R. A. Carbohydr. Res. 2010, 345, 160.

[32] Sakamoto, J. I.; Koyama, T.; Miyamoto, D.; Yingsakmongkon, S.; Hidari, K. I. P. J.; Jampangern, W.; Suzuki, T.; Suzuki, Y.; Esumi, Y.; Hatano, K.; Terunuma, D.; Matsuoka, K. Bioorg. Med. Chem. Lett. 2007, 17, 717.

[33] Klefel, M. J.; Ltzstein, M. Tetrahedron Lett. 1996, 37(40), 7307.

[34] Noel, A.; Delpech, B.; Crich, D. Org. Lett. 2012, 14(5), 1342.

[35] Gantt, R.; Millner, S.; Binkley, S. B. Biochemistry 1964, 3(12), 1952.

[36] Hagiwara, T.; Kijima-Suda, I.; Ido, T.; Ohrui, H.; Tomita, K. Carbohydr. Res. 1994, 263(1), 167.

[37] Burkart, M. D.; Vincent, S. P.; Düffels, A.; Murray, B. W.; Ley, S. V.; Wong, C.-H. Bioorg. Med. Chem. 2000, 8(8), 1937.

[38] Sun, X.-L.; Kanie, Y.; Guo, C.; Kanie, O.; Suzuki, Y.; Wong, C.-H. Eur. J. Org. Chem. 2000, 14, 2643.

[39] Guo, C.-T.; Sun, X.-L.; Kanie, O.; Shortridge, K. F.; Suzuki, T.; Miyamoto, D.; Hidari, K. I.; Wong, C.-H.; Suzuki, Y. Glycobiol 2002, 12(3), 183.

[40] Watts, A. G.; Damager, I.; Amaya, M. L.; Buschiazzo, A.; Alzari, P.; Frasch, A. C.; Withers, S. G. J. Am. Chem. Soc. 2003, 125(25), 7532.

[41] Buchini, S.; Buschiazzo, A.; Withers, S. G. Angew. Chem., Int. Ed. 2008, 47(14), 2700.

[42] Chokhawala, H. A.; Cao, H.; Yu, H.; Chen, X. J. Am. Chem. Soc. 2007, 129, 10630.

[43] Tsai, C.-S.; Yen, H.-Y.; Lin, M.; Tsai, T.; Wang, S.-Y.; Huang, W.; Hsu, T.-L.; Cheng, Y.-S.-E.; Fang, J.-M.; Wong, C.-H. Proc. Natl. Acad. Sci. U. S. A. 2013, 110(7), 2466.

[44] Burkart, M.; Zhang, Z.; Hung, S.; Wong, C.-H. J. Am. Chem. Soc. 1997, 119, 11743.

[45] Rillahan, C. D.; Antonopoulos, A.; Lefort, C. T.; Sonon, R.; Azadi, P.; Ley, K.; Dell, A.; Haslam, S. M.; Paulson, J. C. Nat. Chem. Biol. 2012, $8(7), 661$.

[46] Hader, S.; Watts, A. G. Carbohydr. Res. 2013, 374, 23.

[47] Rudrawar, S.; Dyason, J. C.; Maggioni, A.; Thomson, R. J.; Itzstein, M. Bioorg. Med. Chem. 2013, 21(16), 4820.

[48] Rudrawar, S.; Pascolutti, M.; Bhatt, B.; Thomson, R.; Itzstein, M. Tetrahedron Lett. 2013, 54, 1198.

[49] Zhang, J.; Xu, W.-F. Chin. J. Pharm. 2005, 36(10), 643 (in Chinese).

(张杰, 徐文方, 中国医药工业杂志, 2005, 36(10), 643.)

[50] Zhu, X.-B.; Wang, M.; Wang, S.-Z.; Yao, Z.-J. Tetrahedron 2012, $68,2041$.

[51] Nishino, R.; Ikeda, K.; Hayakawa, T.; Takahashi, T.; Suzuki, T.; Sato, M. Bioorg. Med. Chem. 2011, 19, 2418.
[52] Ikeda, K.; Sato, K.; Kitani, S.; Suzuki, T.; Maki, N.; Suzuki, Y.; Sato, M. Bioorg. Med. Chem. 2006, 14, 7893.

[53] Ikeda, K.; Sato, K.; Nishino, R.; Aoyama, S.; Suzuki, T.; Sato, M. Bioorg. Med. Chem. 2008, 16, 6783.

[54] Ye, D. J.; Li, J.; Zhang, J.; Liu, H.; Jiang, H. L. Tetrahedron Lett. 2007, 48, 4023.

[55] Ye, D.; Deng, G.; Liu, W.; Zhou, Y.; Feng, E.; Jiang, H.; Liu, H. Tetrahedron Lett. 2008, 64, 6544.

[56] Ye, D.; Shin, W. J.; Li, N.; Tang, W.; Feng, E.; Li, J.; He, P. L.; Zuo, J. P.; Kim, H.; Nam, K. Y.; Zhu, W.; Seong, B. L.; No, K. T.; Jiang, H.; Liu, H. Eur. J. Med. Chem. 2012, 54, 764.

[57] Liu, K.-G.; Yan, S.; Wu, Y.-L., Yao, Z.-J. J. Org. Chem. 2002, 67, 6758.

[58] Liu, K.-G.; Zhou, H.-B.; Wu, Y.-L.; Yao, Z.-J. J. Org. Chem. 2003, $68,9528$.

[59] Gao, Z.-X.; Wang, M.; Wang, S.-Z.; Yao, Z.-J. Org. Lett. 2009, 11, 3678 .

[60] Comb, D. G.; Roseman, S. J. Am. Chem. Soc. 1958, 80, 497.

[61] Kok, G. B.; Campbell, M.; Mackey, B. L.; Itzstein, M. Carbohydr. Res. 2001, 332, 133.

[62] Lin, L.; Tian, X.; Hong, S.; Dai, P.; You, Q.; Wang, R.; Feng, L.; Xie, C.; Tian, Z.; Chen, X. Angew. Chem., Int. Ed. 2013, 52(28), 7266.

[63] Chang, P.; Chen, X.; Smyrniotis, C.; Xenakis, A.; Hu, T.; Bertozzi, C. R.; Wu, P. Angew. Chem., Int. Ed. 2009, 48, 4030.

[64] Bond, M. R.; Zhang, H.; Kim, J.; Yu, S. H.; Yang, F.; Patrie, S. M.; Kohler J. J. Bioconjugate Chem. 2011, 22(9), 1811.

[65] Kragl, U.; Gygax, D.; Ghisalba, O.; Wandrey, C. Angew. Chem., Int. Ed. 1991, 30, 827.

[66] Wu, W. Y.; Jin, B.; Kong, D. C. M.; Itzstein, M. Carbohydr. Res. 1997, 300, 171.

[67] Brossmer, R.; Acher, F.; Debacker, C.; Bertrand, H. O.; Ruivo, R.; Sagné, C.; Pietrancosta, N.; Horst, C. A. J. Biol. Chem. 2012, $287(14), 11489$.

[68] Cao, H.; Muthana, S.; Li, Y.; Cheng, J.; Chen, X. Bioorg. Med. Chem. Lett. 2009, 19, 5869.

[69] Suzuki, K.; Sakamoto, J. I.; Koyama, T.; Yingsakmongkon, S.; Suzuki, Y.; Hatano, K.; Terunuma, D.; Matsuoka, K. Bioorg. Med. Chem. Lett. 2009, 19, 5105.

[70] Suzuki, K.; Koyama, T.; Yingsakmongkon, S.; Suzuki, Y.; Hatano, K.; Matsuoka, K. Bioorg. Med. Chem. 2012, 20, 446.

[71] Johansson, S.; Nilsson, E.; Qian, W.; Guilligay, D.; Crepin, T.; Cusack, S.; Arnberg, N.; Elofsson, M. J. Med. Chem. 2009, 52, 3666.

[72] Ogura, H.; Furuhata, K. Carbohydr. Res. 1986, $158,37$.

[73] Allevi, P.; Anastasia, M.; Costa, M. L.; Rota, P. Tetrahedron: Asymmetry 2011, 22, 338.

[74] Honda, T.; Masuda, T.; Yoshida, S.; Arai, M.; Kobayashi, Y.; Yamashita, M. Bioorg. Med. Chem. Lett. 2012, 12, 1921.

[75] Thomson, R.; Itzstein, M. Carbohydr. Res. 1995, 274, 29.

[76] Kong, D. C. M.; Itzstein, M. Tetrahedron Lett. 1995, 36(6), 957.

[77] Wu, J.-R.; Zhan, X.-B.; Zheng, Z.-Y.; Jia, W. Chin. J. Bioprocess Eng. 2007, 5(1), 20 (in Chinese). (吴剑荣, 詹晓北, 郑志永, 贾薇, 生物加工过程, 2007, 5(1), 20.)

[78] Rockle, I.; Seidenfaden, R.; Weinhold, B.; Muhlenhoff, M.; Gerardy-Schahn, R.; Hildebrandt, H. Dev. Neurobiol. 2008, 68, 1170.

[79] Rutishauser, U.; Acheson, A.; Hall, A. K.; Mann, D. M.; Sunshine, J. Science 1988, 240, 53.

[80] Cipolla, L.; Peri, F.; Airoldi, C. Curr. Med. Chem. Anti-Cancer Agents 2008, 8, 92.

[81] Jennings, H. J.; Roy, R.; Michon, F. J. Immunol. 1985, 134, 2651. 
[82] Morley, T. J.; Withers, S. G. J. Am. Chem. Soc. 2010, 132, 9430.

[83] Weïwer, M.; Chen, C. C.; Kemp, M. M. Eur. J. Org. Chem. 2009, 16, 2611.

[84] Shelke, S. V.; Gao. G. P.; Mesch, S.; Gäthje, H.; Kelm, S.; Ernst, O. S. B. Bioorg. Med. Chem. 2007, 15, 4951.

[85] Yu, H.; Cao, H.; Tiwari, V. K.; Li, Y.; Chen, X. Bioorg. Med. Chem. Lett. 2011, 21, 5037.

[86] Schauer, R. Curr. Opin. Struct. Biol. 2009, 19, 507.

[87] Varki, A. Trends Mol. Med. 2008, 14, 351.

[88] Hedlund, M.; Padler-Karavani, V.; Varki, N. M.; Varki, A. Proc. Natl. Acad. Sci. U. S. A. 2008, 105, 18936.

[89] Inoue, S.; Sato, C.; Kitajima, K. Glycobiol 2010, 20, 752.

[90] Kiefel, M. J.; Chopra, P.; Madge, P. D.; Szyczew, A.; Thomson, R. J.; Grice, I. D.; Itzstein, M. Tetrahedron Lett. 2011, 52, 98.

[91] Powell, L. D.; Sgroi, D.; Sjoberg, E. R.; Stamenkovic, I.; Varki, A. J. Biol. Chem. 1993, 268(10), 7019.

[92] Law, C. L.; Aruffo, A.; Chandran, K. A.; Doty, R. T.; Clark, E. A. J. Immunol. 1995, 155(7), 3368.

[93] Greer, S. F.; Justement, L. B. J. Immunol. 1999, 162(9), 5278.

[94] Fujimoto, M.; Bradney, A. P.; Poe, J. C.; Steeber, D. A.; Tedder, T. F. Immunity 1999, 11(2), 191.

[95] Collins, B. E.; Blixt, O.; DeSieno, A. R.; Bovin, N.; Marth, J. D.; Paulson, J. C. Proc. Natl. Acad. Sci. U. S. A. 2004, 101(16), 6104.

[96] Yadav, R.; Kikkeri, R. Chem. Commun. 2012, 48, 7265.
[97] Patterson, D. M.; Nazarova, L. A.; Xie, B.; Kamber, D. N.; Prescher, J. A. J. Am. Chem. Soc. 2012, 134, 18638.

[98] Abdu-Allah, H. H. M.; Watanabe, K.; Completo, G. C.; Sadagopan, M.; Hayashizaki, K.; Takaku, C.; Tamanaka, T.; Takematsu, H.; Kozutsumi, Y.; Paulson, J. C.; Tsubata, T.; Ando, H.; Ishida, H.; Kiso, M. Bioorg. Med. Chem. 2011, 19, 1966.

[99] Yu, H.; Karpel, R.; Chen, X. Bioorg. Med. Chem. 2004, 12, 6427.

[100] Martin, R.; Witte, K. L.; Wong, C.-H. Bioorg. Med. Chem. 1998, 6, 1283.

[101] Liu J. L. C.; Shen, C. J.; Ichikawa, Y.; Rutan, J. F.; Zapata, C.; Vann, W. F.; Wong, C.-H. J. Am. Chem. Soc. 1992, 114, 3901.

[102] Feng, L.-S.; Hong, S.-L.; Rong, J.; You, Q.-C.; Dai, P.; Huang, R.-B.; Tan, Y.-H.; Hong, W.-Y.; Xie, C.; Zhao, J.; Chen, X. J. Am. Chem. Soc. 2013, 135(25), 9244.

[103] Seeberger, P. H.; Haase, W. C. Chem. Rev. 2000, 100(12), 4349.

[104] Giepmans, B. N.; Adams, S. R.; Ellisman, M. H.; Tsien, R. Y. Science 2006, 312(5771), 217.

[105] Lecuyer, E.; Yoshida, H.; Parthasarathy, N.; Alm, C.; Babak, T.; Cerovina, T.; Hughes, T. R.; Tomancak, P.; Krause, H. M. Cell 2007, 131(1), 174.

[106] Dube, D. H.; Bertozzi, C. R. Curr. Opin. Chem. Biol. 2003, 7, 616.

[107] Laughlin, S. T.; Baskin, J. M.; Amacher, S. L.; Bertozzi, C. R. Science 2008, 320(5876), 664. 